\title{
Chapter 28 \\ Coping with Wheat Quality in a Changing Environment: Proteomics Evidence for Stress Caused by Environmental Changes
}

\author{
Gérard Branlard, Véronique S. Lesage, Emmanuelle Bancel, Pierre Martre, \\ Benoit Méleard, and Larbi Rhazi
}

\begin{abstract}
High temperatures (HTs) during grain filling adversely impact grain yield and its end-use quality for wheat. HTs strongly reduce the expression of major enzymes associated with starch synthesis, whereas enzymes associated with defence against stress and protein folding are dramatically increased. Using proteomics tools, the effect of different temperature regimes on storage protein (SP) accumulation was investigated. HT significantly decreased the quantity per grain of individual gliadin and glutenin spots, but at maturity the ratio of gliadin to glutenin was not modified. HT during grain filling strongly reduced starch accumulation, modified the size distribution of starch granules, and to a much lesser extent, reduced the quantity of total proteins per grain. The aggregation and polymerisation of SP was investigated using asymmetric flow field flow fractionation. Previous analyses of near-isogenic hard/soft lines showed that characteristics of glutenin polymers were significantly influenced by puroindoline alleles (Pina-Dla and $-D 1 b$ ), and proteomics analysis showed that a typical mechanism of unfolded protein response occurs in ER, resulting from stress during protein accumulation. Effects of alleles encoding puroindolines, HMW-GS and LMW-GS, and temperature during grain development on glutenin polymer characteristics, dough rheological properties, and bread loaf volume were investigated for 40 cultivars grown in six environments in France. A difference of only $2{ }^{\circ} \mathrm{C}$ in average daily air temperature between locations during the grain-filling period resulted in increased molecular mass of the glutenin
\end{abstract}

\footnotetext{
G. Branlard $(\triangle) \bullet$ V.S. Lesage $\bullet$ E. Bancel $\bullet$ P. Martre

INRA, UMR1095 Genetics, Diversity and Ecophysiology of Cereals,

5 chemin de Beaulieu, Clermont-Ferrand F-63 039, France

Blaise Pascal University, UMR1095 Genetics,

Diversity and Ecophysiology of Cereals, Aubière F-63173, France

e-mail: branlard@clermont.inra.fr

B. Méleard

Arvalis-Institut du Végétal, Boigneville F91720, France

L. Rhazi

Institut Polytechnique LaSalle Beauvais, Beauvais F60026, France
} 
polymers and dough tenacity, but decreased dough extensibility and bread loaf volume. To compensate these adverse effects, some solutions are suggested.

Keywords Dough properties $\bullet$ Endosperm $\bullet$ Gliadins $\bullet$ Glutenins $\bullet$ Glutenin polymers $\bullet$ Heat stress $\bullet$ Proteomics $\bullet$ Starch $\bullet$ Temperature

\section{High Temperature Is a Major Factor Affecting Wheat Production}

Cereals and particularly winter crops are influenced by ongoing climate changes. Drought may strongly affect plant physiological development resulting in fewer fertile ears per plant and fewer grains per spike. Among abiotic stresses, heat stress, particularly during grain filling, is the main factor responsible for a reduction in grain weight, and hence in yield (Jenner 1994; Ottman et al. 2012). High temperatures when storage compounds are accumulating in the grain are one of the main factors influencing wheat end-use value (Wardlaw and Wrigley 1994). Although a gradual rise in daily maximum temperatures causes less damage to grain compounds than a sudden temperature rise during grain filling, wheat cultivars may also respond differently to high temperatures (Stone and Nicholas 1994; Stone et al. 1997). Whatever the cultivar studied, high temperatures have been shown to shorten the effective grain filling period, and may significantly increase protein concentration because of more dramatic effect on starch than on protein accumulation (Altenbach et al. 2003; Blumenthal et al. 1991a, b, 1995; Corbellini et al. 1998; Triboï et al. 2003). Many studies have addressed the responses of wheat grain to high temperatures during grain filling using either transcriptomics tools (Altenbach and Kothari 2004; Altenbach et al. 2007; Hurkman et al. 2003; Perrotta et al. 1998) or proteomics approaches (Hurkman et al. 2009; Laino et al. 2010; Majoul et al. 2003, 2004; Majoul-Haddad et al. 2013; Skylas et al. 2002; Vensel et al. 2005). In the present study, major events occurring in the wheat endosperm in response to high temperatures during grain filling were investigated using proteomics approaches.

The present report describes major variations in individual storage proteins (SP), metabolic enzymes and proteins involved in carbohydrate metabolism and glutenin polymer formation in response to HT, based on three experiments carried out under controlled conditions and one multi-location field trial in France.

\section{Experiments Used for Heat Stress Studies}

Three experiments were carried out at INRA Clermont-Ferrand $\left(45^{\circ} 46^{\prime} \mathrm{N}, 03^{\circ}\right.$ 09'E, 329 m a.s.l.) in the Crop Climate Control and Gas Exchange Measurement (C3-GEM) platform (Triboi et al. 1996) to analyze wheat (Triticum aestivum L.) grain responses to heat treatments. Crops were grown outside in $2-\mathrm{m}^{2}$ containers 
$0.5 \mathrm{~m}$ deep. Seeds were sown in mid-November at a density of 578 seeds $\mathrm{m}^{-2}$. Crops were watered and fertilized to avoid any growth limitation with an objective of grain protein concentration of $12.5 \%$. At anthesis the containers were transferred under transparent enclosures under natural light in the C3-GEM platform and exposed to the following conditions:

Experiment 1 Moderate high temperature (MHT) treatment. Cultivars Arche and Tamaro were maintained at $23{ }^{\circ} \mathrm{C} / 1{ }^{\circ} \mathrm{C}$ (day/night) for the control and at $28{ }^{\circ} \mathrm{C} / 15$ ${ }^{\circ} \mathrm{C}$ for the MHT treatment. Grains were sampled at seven stages from $163{ }^{\circ} \mathrm{C}$ days (cumulative degree-days after anthesis) to $781{ }^{\circ} \mathrm{C}$ days (physiological maturity) after anthesis.

Experiment 2 Very high temperature (VHT) treatment. Cultivar Thésée was maintained at $18{ }^{\circ} \mathrm{C} / 10{ }^{\circ} \mathrm{C}$ for the control treatment and $34{ }^{\circ} \mathrm{C} / 10{ }^{\circ} \mathrm{C}$ for the VHT treatment. Results of proteomics analysis of endosperm responses to VHT based on this experiment for total proteins were reported by Majoul et al. (2003) and for albuminsglobulins (AG) by Majoul et al. (2004).

Experiment 3 Heat shock (HS) treatment. Cultivar Récital was grown with a day/ night temperature of $18{ }^{\circ} \mathrm{C} / 10{ }^{\circ} \mathrm{C}$. One container was subjected to 4-h periods at 38 ${ }^{\circ} \mathrm{C}$ for four consecutive days (HS treatment) between 300 and $400{ }^{\circ} \mathrm{C}$ days after anthesis. Total proteins and AG were analysed just before and 1, 8, and 26 (ripeness) days after HS were applied. Proteomics analysis of grain responses to HS based on this experiment was reported by Majoul-Haddad et al. (2013).

The Multi-location Field Trial A total of 68 genetically diverse wheat cultivars provided by INRA and by 11 wheat private breeding companies were grown in three locations in France in 2009 and 2010. The cultivars were grown in conventional conditions with full mineral supply and fungicide protection. The aim of the multi-location trial was to better understand the genetic and environmental factors which influence three parameters: dough tenacity $(\mathrm{P})$ and extensibility $(\mathrm{L})$ and bread loaf volume. The grain composition and quality characteristics of 240 samples (40 cultivars $\times 6$ environments) were analyzed.

\section{Main Proteomics Responses of Developing Wheat Grain to High Temperature}

\section{Major Impacts of High Temperature on Energy Metabolism and Starch Synthesis}

In all experiments average single grain mass was significantly reduced with the temperature treatments. In experiment 3 , the HS treatment $\left(38^{\circ} \mathrm{C}\right.$ for $4 \mathrm{~h}$ on 4 consecutive days) caused a $25 \%$ reduction in single grain dry mass at maturity (26 days after the HS period; Majoul-Haddad et al. 2013). Although here the amount of starch per grain was not determined, previous studies have shown that reduced grain 
masses in response to elevated temperature results from reduced accumulation of starch (e.g. Altenbach et al. 2003; Hurkman et al. 2009). Most proteomics studies have reported a significant decrease in the amount of the small subunits and/or large subunits of the ADP glucose pyrophosphorylase (AGPase, also named glucose 1 phosphate adenyl transferase), the enzyme that catalyses the first committed step in starch biosynthesis pathway, in response to elevated temperature. In experiment 2 , at maturity, the AGPase was reduced by $50 \%$ for VHT compared to control. However, in experiment 3, no significant decrease was observed in response to HS. Surprisingly, the abundance of enzymes involved in amylopectin synthesis (starch synthase SSI and SSII; starch branching enzymes SBE I and SBE II; and starch debranching enzyme) was not reduced by elevated temperatures. The abundance of some of these enzymes was slightly, but not consistently, decreased for HS compared to control. This discrepancy may be associated with the specific procedure required for the extraction of these enzymes, a procedure that is rarely performed in proteomic studies (Bancel et al. 2010). The abundance of granule-bound starch synthase (GBSS I) was increased fivefolds for HT compared to control, in good agreement with the higher amylose content previously observed for grain exposed to elevated temperature (Shi et al. 1994).

Transcriptomic and proteomic analyses of grain exposed to HT revealed an increase of transcripts and proteins involved in glycolysis. Here, the amount of phosphohexose isomerase, aldolase, triose phosphate isomerase, glyceraldehyde phosphate dehydrogenase, phosphoglycerate kinase, and enolase increased in response to VHT (experiment 2), or were transiently present in response to HS (experiment 3). Grains exposed to HT require more energy (ATP) as evidenced by the higher abundance of the glycolysis enzymes, reducing the amount of glucose available for starch accumulation. The $\beta$-amylase present in the late stage of grain filling (Hurkman et al. 2009) was also reported to have increased threefolds at maturity for VHT compared to control in experiment 2 . The over expression of $\beta$-amylase, usually found in germinating grain, occurs to provide the endosperm with energy. The above findings indicate that starch accumulates less due to higher glycolysis and is also partly hydrolysed by $\beta$-amylase, probably to provide the endosperm cells with energy. In HT samples, the consequences were a reduced volume of B and C starch granules and correlatively an increased proportion of A granules (Table 28.1).

Table 28.1 Percent volume of large (A-type, diameter $>15 \mu \mathrm{m}$ ), intermediate (B-type, diameter $=5-15 \mu \mathrm{m})$ and small $(\mathrm{C}$-type, diameter $<5 \mu \mathrm{m})$ starch granules determined using laser granulometry for mature grains of $\mathrm{cv}$. Récital grown under normal temperature regime (control) or exposed to $38{ }^{\circ} \mathrm{C}$ for $4 \mathrm{~h}$ on for 4 consecutive days (heat shock, experiment 3 )

\begin{tabular}{l|l|l|l}
\hline & Percent volume (\%) & C \\
\hline Temperature treatment & A & B & $1.2 \pm 0.01$ \\
\hline Control & $71.0 \pm 0.3$ & $27.8 \pm 0.3$ & $1.0^{\mathrm{a}} \pm 0.01$ \\
\hline Heat shock & $78.9^{\mathrm{a}} \pm 0.2$ & $20.1^{\mathrm{a}} \pm 0.2$ & \\
\hline
\end{tabular}

${ }^{a}$ Significantly different from control at $P<0.0001$

Data are means $\pm 1 \mathrm{SD}$ for $n=3$ independent replicates 


\section{Synthesis of Wheat Storage Proteins Is Not Drastically Affected by High Temperature}

MHT treatment (experiment 1) caused variation in the amount of only few individual SP of cv. Arche: two $\omega$-gliadins were increased, whereas one $\gamma$-gliadin and one LMW-GS were reduced. No HMW-GS was affected by MHT. However, MHT resulted in a significant decrease in the amount of SP expressed in micrograms of protein per grain, as only $65.7 \%$ and $65.5 \%$ of the total amount of gliadin and glutenin, respectively, were accumulated at maturity, compared to control.

VHT treatment increased (2- to 27-folds) the amount of only three $\alpha$-gliadins in the mature grain of cv. Thésée (Majoul et al. 2003) and HMW-GS and LMW-GS were not modified by the treatment.

HS treatment affected the kinetics of accumulation of 13 SPs. Ten of these proteins still differed significantly at maturity: four $\alpha$-gliadins, two $\gamma$-gliadins, and two LMW-GS were decreased and one $\beta$-gliadin and one $\omega$-gliadin were increased compared to control. In an early study, one HMW-GS encoded by Glu-Al significantly increased when expressed as a percentage volume (Branlard et al. 2008). Reassessing the analysis of all 2D gel images failed to confirm this higher abundance when expressed in micrograms of protein per grain (Majoul-Haddad et al. 2013), again providing evidence that the accumulation of HMW-GS was not affected by the HS treatments.

In addition to the remarkable stability in abundance exhibited by HMW-GS in response to HT and considering that more than $80 \mathrm{SP}$ spots were detected on 2D gels whatever the cultivar analysed, it should be noted that only few SP were influenced by HT treatments. In all three experiments, the kinetics of individual SP was analysed. During grain filling, the ratio of gliadin to glutenin was different at one (640 ${ }^{\circ} \mathrm{C}$ days after anthesis) out of seven sampling stages for $\mathrm{cv}$. Arche (medium quality wheat) whereas no difference was found for cv. Tamaro (high quality wheat). At maturity, no difference between heat exposed and control grains were detected for the ratio of gliadin to glutenin. Altogether, the amount of individual SP during grain filling demonstrated remarkable stability in response to HT treatments, indicating the existence of regulatory mechanisms, which could differ between cultivars. This underlines the need for a better understanding of the regulatory gene networks involved in SP synthesis when breeding future high quality cultivars.

\section{Glutenin Polymers Are Strongly Impacted by High Temperature}

The molecular mass of glutenin polymers was previously shown to be significantly influenced by grain hardness (Lesage et al. 2011). Further investigations (Lesage et al. 2012) of the puroindoline function provided clear evidence that the endoplasmic reticulum (ER) is the site of the unfolded protein response (UPR), a phenomenon first described in animal cells (Schröder and Kaufman 2005). Protein folding is 
known to be inhibited by several factors including excess proteins in ER, temperature, magnetic field, and electric field. It was thus necessary to investigate the influence of the characteristics of the polymers for cultivars of different genetic origin and grown in different locations. The polymer characteristics (molecular mass Mw2, polydispersity index Mw2/Mn2 and radius of polymers Rw2) of the 240 samples from the multi-location field trial were evaluated using asymmetric flow field flow fractionation (AFFFF; Lemelin et al. 2005). Glutenin alleles and puroindoline alleles, as well as their interactions were shown to significantly influence Mw2, which, unexpectedly, varied from $5 \times 10^{6}$ to $49 \times 10^{6}$ Da (Lesage et al. 2013). In addition, it was shown that the sum of daily mean air temperatures for June and July (i.e. during the grain filling period; SumT-JuJy), differentially affected Mw2 in the three hardness classes (hard, medium and soft). A difference of $110{ }^{\circ} \mathrm{C}$ in SumTJuJy was found across the six environments, which was correlated with an increase of Mw2 from $10 \times 10^{6}$ to $20 \times 10^{6}$ Da in hard cultivars and from $13 \times 10^{6}$ to $33 \times 10^{6}$ $\mathrm{Da}$ in soft cultivars. Partial least square regression used to explain Mw2 with several grain characteristics including glutenin and puroindoline alleles and SumT-JuJy, provided clear evidence for the major influence of SumT-JuJy on phenotypic variations in polymer characteristics (Fig. 28.1a). Why does temperature have such a strong effect on the characteristics of glutenin polymers? All the proteomics studies on grain response to HT reported that two to four of the redox enzymes ascorbate dismutase, ascorbate peroxidase, dehydroascorbate reductase, and glutathione reductase were increased by HT. This, together with evidences of UPR, suggests that grains exposed to HT undergo sever oxidative stresses causing higher polymer mass.

\section{Variations in Dough Properties Resulting from High Temperature}

The rheological properties of dough and bread loaf volume measured on the 240 samples revealed that SumT-JuJy had a strong impact on these important quality parameters. Indeed, the increase of the molecular mass of glutenin polymers in response to temperature was correlated with an increase in dough tenacity (Fig. 28.1b) and a decrease in both dough extensibility (Fig. 28.1c) and loaf volume (Fig. 28.1d). The standardized PLS coefficients attributed to SumT-JuJy were among the highest in explaining phenotypic variations in dough tenacity, dough swelling, and bread loaf volume. Dough tenacity was, as expected, positively influenced by grain hardness, Glu-B1 7-8, Glu-Dl 5-10 and Glu-B3c, and negatively influenced by GluB1 6-8, Glu-B1 6.1-22, Glu-D1 2-12, Glu-B3b'(bp in Fig. 28.1) but surprisingly, also negatively influenced by Glu-A1 $2^{*}$ and Glu-B1 17-18. Dough extensibility increased with grain protein content (GPC) as is usually the case, but also increased with some glutenin alleles like Glu-A1 2*, Glu-B1 17-18. The glutenin subunits Glu-D1 5-10 were negatively correlated with dough extensibility and Glu-D1 2-12 

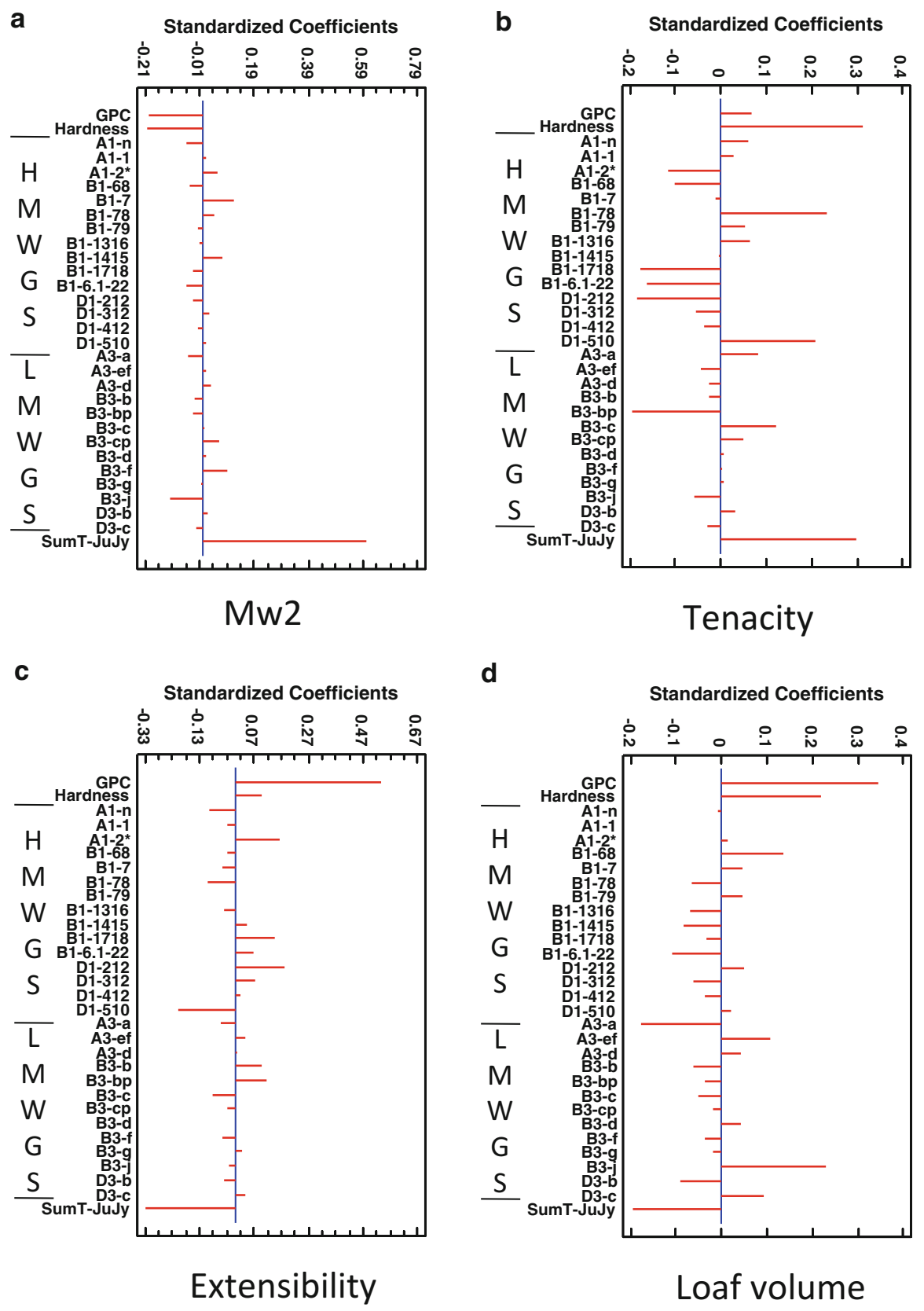

Fig. 28.1 Partial least square regression performed to explain the molecular mass of gluten in polymers, Mw2 (a), dough tenacity (b) and extensibility (c), and bread loaf volume (d): the negative (left horizontal bar) and positive (right horizontal bar) standardized coefficients were computed for grain protein concentration (GPC), grain hardness (Hardness), HMW-GS and LMW-GS alleles, and the sum of mean daily temperature during the grain filling period (SumT- JuJy: June and July) 
was positively correlated with dough extensibility. In addition to the well-known positive effect of GPC and grain hardness on bread loaf volume, the standardized coefficients attributed to glutenin alleles clearly evidenced that the molecular mass of glutenin polymers had a negative impact on loaf volume. This result was supported by the positive correlation between loaf volume and glutenin alleles known to have a very negative effect on dough rheological properties (Glu-Bl 6-8, Glu$A 3 e f$, and Glu-B3j).

\section{Some Proposals to Cope with Wheat Quality in a Changing Environment}

High temperatures during grain filling have a more pronounced effect on the amount of starch than on the amount of protein accumulated per grain. All genetic factors associated with higher starch accumulation including light saturated photosynthesis, awned ears, and prolonged photosynthesis in grain peripheral layers, must be regarded as potentially favorable for maintaining higher amounts of starch per grain during HT events (reviewed by Cossani and Reynolds 2012). The influence of HT on the genetic regulation of major starch enzymes and particularly AGPase and $\beta$-amylase should now be investigated in wheat to identify potential markers for breeders. Whatever the potential benefit of using those markers, determination of the test weight, negatively impacted by HT during grain filling, is the easiest way to phenotype wheat progenies capable of completing grain filling even in a HT environment.

Only few individual SPs are influenced by HT or heat shock during the linear phase of grain filling. The ratio of gliadin to glutenin was not significantly affected by the HT treatments used here. However, higher temperatures (daily maximum $>35{ }^{\circ} \mathrm{C}$ ) have been reported to increase the gliadin to glutenin ratio (Blumenthal et al. 1995). Although individual SP may not be affected, we showed that daily maximum temperature lower than $35^{\circ} \mathrm{C}$ can strongly increase the molecular mass of the glutenin polymers. The resulting effect is to increase dough tenacity and decrease extensibility. This may explain why, in the last decade, the ratio of tenacity to extensibility has often been reported to have increased in many countries where the alveograph test is used. To tackle such quality consequences, it is advisable not to discard alleles previously associated with dough extensibility like Glu-A1 2*, Glu-B1 17-18 and Glu-D1 2-12. The influence of SP diversity on the molecular mass of glutenin polymers and wheat properties also needs to be reassessed in relation with puroindoline alleles, as judiciously proposed by Ikeda et al. (2013). The molecular regulation of redox enzymes such as dehydroascorbate reductase, glutathione reductase, and glutathione transferase also need to be investigated to improve the stability of the molecular mass of glutenin polymers in HT environments. 
Acknowledgments François Xavier Oury (INRA Clermont Ferrand) and breeders from the wheat breeding companies: Caussade Semences, Florimond Desprez, Lemaire Deffontaines, Limagrain, Momont et Fils, R2N, Saaten-Union Recherche, Secobra Recherches, Serasem, Syngenta and Unisigma, are gratefully acknowledged for their active participation in the multilocation field trials and in studies on tenacity/extensibility and bread loaf volume.

Open Access This chapter is distributed under the terms of the Creative Commons Attribution Noncommercial License, which permits any noncommercial use, distribution, and reproduction in any medium, provided the original author(s) and source are credited.

\section{References}

Altenbach SB, Kothari KM (2004) Transcript profiles of genes expressed in endosperm tissue are altered by high temperature during wheat grain development. J Cereal Sci 40:115-126

Altenbach SB, DuPont FM, Kothari KM et al (2003) Temperature, water and fertilizer influence the timing of key events during grain development in a US spring wheat. J Cereal Sci 37:9-20

Altenbach SB, Kothari KM, Tanaka CK, Hurkman WJ (2007) Genes encoding the PR-4 protein wheatwin are developmentally regulated in wheat grains and respond to high temperature during grain fill. Plant Sci 173:135-143

Bancel E, Rogniaux H, Debiton C et al (2010) Extraction and proteome analysis of starch granuleassociated proteins in mature wheat kernel (Triticum aestivum L.). J Proteome Res 9:3299-3310

Blumenthal CS, Bekes F, Batey IL et al (1991a) Interpretation of grain quality results from wheat variety trials with reference to high temperature stress. Aust J Agric Res 42:325-334

Blumenthal CS, Batey IL, Bekes F et al (1991b) Seasonal changes in wheat-grain quality associated with high temperatures during grain filling. Aust J Agric Res 42:21-30

Blumenthal CS, Bekes F, Gras PW et al (1995) Identification of wheat genotypes tolerant to the effects of stress on grain quality. Cereal Chem 72:539-544

Branlard G, Bancel E, Majoul T, Martre P (2008) Proteomics evidence of quality stresses caused by changing environment. In: Proceedings of the 11 th international wheat genetics symposium, Brisbane

Corbellini M, Mazza L, Ciaffi M et al (1998) Effect of heat shock during grain filling on protein composition and technological quality of wheats. Euphytica 100:147-154

Cossani CM, Reynolds MP (2012) Physiological traits for improving heat tolerance in wheat. Plant Physiol 160:1710-1718

Hurkman WJ, McCue KF, Altenbach SB et al (2003) Effect of temperature on expression of genes encoding enzymes for starch biosynthesis in developing wheat endosperm. Plant Sci 164:873-881

Hurkman WJ, Vensel WH, Tanaka CK et al (2009) Effect of high temperature on albumin and globulin accumulation in the endosperm proteome of the developing wheat grain. J Cereal Sci 49:12-23

Ikeda TM, Rogers WJ, Branlard G, et al. (2013) Proposal of international gluten research group. In: Proceedings of the 12th international wheat genetics symposium, Yokohama

Jenner CF (1994) Starch synthesis in the kernel of wheat under high temperature conditions. Aust J Plant Physiol 21:791-806

Laino P, Shelton D, Finnie C et al (2010) Comparative proteome analysis of metabolic proteins from seeds of durum wheat (cv. Svevo) subjected to heat stress. Proteomics 10:2359-2368

Lemelin E, Branlard G, Salvo L et al (2005) Bread making stability of wheat flours: relation between mixing properties and molecular weight distribution of polymeric glutenins. J Cereal Sci 42:317-326 
Lesage VS, Bouchet B, Rhazi L et al (2011) New insight into puroindoline function inferred from their subcellular localization in developing hard and soft near-isogenic endosperm and their relationship with polymer size of storage proteins. J Cereal Sci 53:231-238

Lesage VS, Merlino M, Chambon C et al (2012) Proteomes of hard and soft near-isogenic wheat lines reveal that kernel hardness is related to the amplification of a stress response during endosperm development. J Exp Bot 63:1001-1011

Lesage VS, Rhazi L, Aussenac T, et al. (2013) Effects of HMW- \& LMW-glutenins and grain hardness on size of gluten polymers. In: He Z, Wang D (eds) Wheat Gluten 2012, Proceedings of the 11th international wheat gluten workshop, Beijing, pp 200-205

Majoul T, Bancel E, Triboi E et al (2003) Proteomic analysis of the effect of heat stress on hexaploid wheat grain: characterization of heat-responsive proteins from total endosperm. Proteomics 3:175-183

Majoul T, Bancel E, Triboi E et al (2004) Characterization of heat-responsive proteins from nonprolamins fraction. Proteomics 4:505-513

Majoul-Haddad T, Bancel E et al (2013) Effect of short heat shocks applied during grain development on wheat (Triticum aestivum L.) grain proteome. J Cereal Sci 57:486-495

Ottman MJ, Kimball BA, White JW, Wall GW (2012) Wheat growth response to increased temperature from varied planting dates and supplemental infrared heating. Agron J 104:7-16

Perrotta C, Treglia AS, Mita G et al (1998) Analysis of mRNAs from ripening wheat seeds: the effect of high temperature. J Cereal Sci 27:127-132

Schröder M, Kaufman RJ (2005) The mammalian unfolded protein response. Annu Rev Biochem 74:739-789

Shi YC, Seib PA, Bernardin JE (1994) Effects of temperature during grain-filling on starches from six wheat cultivars. Cereal Chem 71:369-383

Skylas DJ, Cordwell SJ, Hains PG et al (2002) Heat shock of wheat during grain filling: proteins associated with heat-tolerance. J Cereal Sci 35:175-188

Stone PJ, Nicholas ME (1994) Wheat cultivars vary widely in their responses of grain yield and quality to short periods of post-anthesis heat stress. Aust J Plant Physiol 21:887-900

Stone PJ, Gras PW, Nicolas ME (1997) The influence of recovery temperature on the effects of a brief heat shock on wheat. III. Grain protein composition and dough properties. J Cereal Sci 25:129-141

Triboï E, Triboï-Blondel AM, et al (1996) Experimental device for studying post-anthesis canopy functioning in relation to grain quality. In: Proceedings of the 4th European society of agronomy congress, Veldhoven and Wageningen, 7-11 July 1996, pp 68-69

Triboï E, Martre P, Triboï-Blondel AM (2003) Environmentally-induced changes of protein composition for developing grains of wheat are related to changes in total protein content. J Exp Bot 54:1731-1742

Vensel WH, Tanaka CK, Cai N et al (2005) Developmental changes in the metabolic protein profiles of wheat endosperm. Proteomics 5:1594-1611

Wardlaw IF, Wrigley CW (1994) Heat tolerance in temperate cereals - an overview. Aust J Plant Physiol 21:695-703 